Journal of Information and Communications Engineering (JICE), 6 (2): 404-410, December 31, 2020

e-ISSN: 2432-5465, p-ISSN: 2186-9162

Applied Science and Computer Science Publications

\title{
Formation of efficient and inefficient social convention driven by conformity bias
}

\author{
Akira Masumi and Takashi Sato
}

\begin{abstract}
Social conventions govern social behavior in many ways, ranging from left- and right-hand traffic to greetings. We sometimes find that inefficient social conventions, such as bullying in a class, are spontaneously formed, where almost all the people in the group are at a disadvantage. Although such a convention can be disadvantageous for all the people in the group, why are those conventions formed and continue to be maintained? A conformity bias, the behavioral tendency with which people take an action that a majority of the group take, can be one of the key ingredients of this phenomenon. In this study, we investigated the impact of conformity bias and an individual's trial-and-error learning on the spontaneous formation of social conventions. Analyzing the stationary states of the dynamics and the tipping points at which the dynamics are divided into one converging to the efficient and the other converging to the inefficient convention, we found that by increasing the extent of conformity bias, an inefficient convention tends to be formed. On the other hand, an individual's trial-and-error learning can suppress conformity bias and promote the formation of efficient conventions.
\end{abstract}

Keywords: Social convention; Conformity bias; Positive externality; Reinforcement learning model; Tipping points

\section{INTRODUCTION}

$\mathrm{S}_{\mathrm{w}}^{\mathrm{o}}$ ocial conventions govern our daily behavior in many ways. For example, when we drive a car in Japan, we keep on the left-hand side of the road as others do. If we drive on the right-hand side, we will surely have an accident; therefore we should keep driving on the lefthand side rule. Such explicit or implicit rules governing social behavior are called social conventions [1][2].

Notably, many of these conventions are spontaneously formed for various historical reasons. In the example of a driving lane, drivers stay to the left in Japan but on the right in the United States and other countries. The side of the road on which a car drives does not really matter if all drivers follow the same rule. Achieving an efficient traffic flow, that is, avoiding traffic accidents, is the most important thing. In addition, how such conventions are spontaneously formed is an interesting question that needs to be addressed.

By contrast, we sometimes find that inefficient social conventions are formed where people in a group cannot escape from such situations despite suffering a disadvantage [3][4]. Bullying can be considered an example of such a situation. When bullying occurs in a class, the student who is bullied suffers a disadvantage.
In addition, students who are complicit in bullying may also suffer if they are unwilling to do so, for example, being forced to join the bully. Even for the bully, leading a bullying is potentially disadvantageous; because he/she may be severely punished by his/her teacher.

According to the discussion above, the class or group of students dealing with bullying is inefficient compared to a group without any bullying, where no one is suffering a disadvantage. Nevertheless, why are these conventions formed and maintained?

Recent studies showed that conformity bias can be a key ingredient in the formation of inefficient social conventions [2][3][5]. The conformity bias is a behavioral tendency in which people take action that a majority of the group takes. This bias can promote the formation of the social convention because of a positive feedback effect on the number of people choosing a certain action; the more people who take a certain action, the more that people taking the action increases.

Furthermore, it is known that conformity bias varies with age; previous studies showed that younger people display higher levels of conformity bias than older people [6][7]. This result implies that conformity bias may play a significant role in the formation of an 
inefficient social convention characteristic of younger people, such as bullying.

As a theoretical approach to the formation of an efficient and inefficient convention, previous studies proposed a model of the utility of an individual that incorporates the conformity bias as a "social effect" from the group [5][8][9][10]. In this model, the utility of individuals for a certain option is composed of the intrinsic utility gained from the option and popularity, representing how the option is chosen by other individuals in the group. This formulation of the utility allows us to analyze the qualitative and quantitative effects of conformity bias on the formation of efficient and inefficient conventions.

Meanwhile, behavioral experiments assuming a game-theoretic situation among individuals showed that human participants can spontaneously form a convention through repeated interaction [11][12]. In addition, the behavioral data of the participants are well fitted with a reinforcement learning model in which individuals learn an action that maximizes his/her expected reward through trial and error [13].

The conformity bias and the individual's trial-anderror learning can play an opposite role in the formation of an inefficient convention. The conformity bias may lead to inefficient convention if the individuals in the group who take an inefficient option become the majority. This possibility increases when the extent of an individual's conformity bias becomes greater. On the other hand, an individual's trial-and-error learning promotes the formation of an efficient convention. Although the mutual effect of these two factors on the formation of a convention can be critical, as far as we know, only a few studies have focused on such a phenomenon. Can an individual's trial-and-error learning overcome conformity bias? If that is the case, under what conditions can it occur?

In this study, we investigated the impact of conformity bias and an individual's trial-and-error learning on the formation of the convention. As a method, we construct a game-theoretic model of the spontaneous formation of the convention. By analyzing the dynamics and stationary states of the model, we explore how conformity bias and an individual's learning mutually interact with each other and contribute to the formation of the convention.

\section{METHOD}

\section{A. Reward function}

In our model, the individuals play a repeated game, defined as follows. In each stage of the game, the players choose one of two options $j \in\{X, Y\}$ and gain a reward following a reward function defined later. We assume a positive externality in a reward function: the more players who choose the same option as player $i$ 's choice, the more player $i$ receives a higher reward. Let $N$ be the number of players. $n_{j}(t)$ is the number of players who choose an option $j$ at time $t$, where $n_{X}(t)+n_{Y}(t)=$ $N$. Then, we define the frequency of the players in the group choosing option $j$ at time $t$ as $f_{j}(t):=n_{j}(t) / N$. Based on these, we define a reward function as a linear function of the frequency at time $t$ as follows:

$r_{X}(t)=X_{\max } f_{X}(t)$

$r_{Y}(t)=Y_{\max }\left(1-f_{X}(t)\right)$,

where $X_{\max }$ and $Y_{\max }$ are positive and represent the maximum values of rewards for options $X$ and $Y$, respectively as shown in Figure 1.

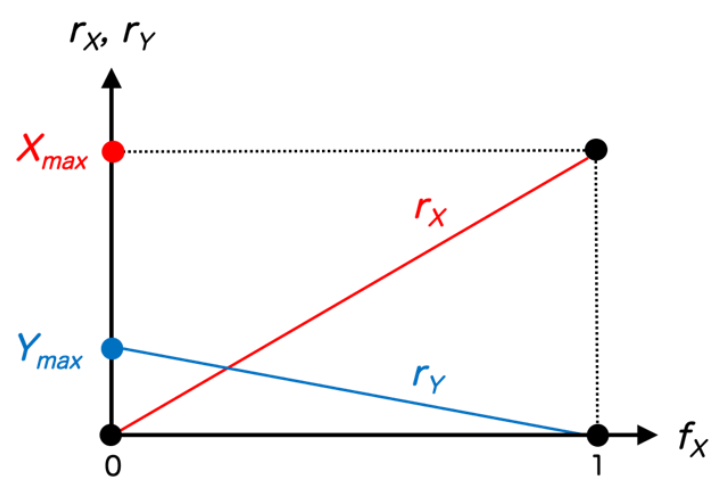

Fig. 1. Reward function of present model. Horizontal axis represents frequency of option $X$, and vertical axis represents reward for each option, $r_{X}$ and $r_{Y} . X_{\max }$ and $Y_{\max }$ are maximum values of rewards for each option.

Considering the possibility of formation of both an efficient and inefficient social conventions, we assume $X_{\max }>Y_{\max } ; X$ is a relatively "good" option that yields higher rewards than $Y$ when all players choose the same option, i.e., $f_{X}(t)=0$ or 1 . We define the relative "badness" of option $Y$ as follows:

$\theta:=\frac{Y_{\max }}{X_{\max }}$

In this study, we set $X_{\max }$ to 1 . Then, the range of $\theta$ is given as $\theta \in(0,1)$. 
In a stage game defined by $N, j \in\{X, Y\}$, and the reward function, the players play an infinitely repeated game. As a result of playing this repeated game, it is expected that dynamics will converge to a certain stationary state. The types of frequency $f_{X}(t)$ and dynamics depend on the initial conditions and parameters of the model (explained later). In the present study, we refer to these stationary states as social conventions.

Assuming $X_{\max }>Y_{\max }$, we can see that a combination of choices of all players in which all of them choose $X$ is a unique Pareto efficient combination (see Fig. 1). On the other hand, combinations other than the Pareto efficient are all inefficient in the sense that, in a such combinations, at least one player reduces his/her reward compared with that gained from the case of the Pareto efficient combination. Therefore, as the number of players who choose $Y$ in an emerging social convention increases, social conventions become more inefficient. In this study, we use the terms efficiency and inefficiency in this sense.

\section{B. Biased reward}

We introduce conformity bias to our model with the following assumptions. First, we assume that the players' evaluation of $r_{j}(t)$ is positively biased by the number of players who choose an option $j$. Namely, the more players choose an option $j$, the more they put a high value on option $j$. Then, we assume that this biased evaluation is proportional to the frequency $f_{j}(t)$ at time $t$, and its strength is represented by a conformity bias coefficient $b$. Moreover, we assume that a biased reward is composed of the biased evaluation and the nonbiased reward to option $j$ [8][9][10]. According to the above assumptions, a biased reward $r_{j}^{\prime}(t)$ is defined as:

$r_{X}^{\prime}(t)=(1-b) r_{X}(t)+b f_{X}(t)$

$r_{Y}^{\prime}(t)=(1-b) r_{Y}(t)+b f_{Y}(t)$.

Here, $b \in[0,1]$ is the conformity bias coefficient of each player, which represents the extent to which each player's choice is biased by the choices of the others in the group. If $b>0$, then the players evaluate the reward gained by taking option $j$ higher than a nonbiased reward per Equations (1) and (2), depending on the frequency of the option. Assuming $X_{\max }=1$ and substituting Equations (1)-(3) into (4) and (5) yields the following results:

$r_{X}^{\prime}(t)=f_{X}(t)$

$r_{Y}^{\prime}(t)=\left(1-f_{X}(t)\right)[\theta(1-b)+b]$.

\section{Reinforcement learning model}

In our model, we assume that players try to maximize their expected rewards following a certain adaptive mechanism. As such, we employed a reinforcement learning model. In the reinforcement learning model, a player assigns a value to an option using a weighted sum of rewards gained by taking the option. They choose an option based on probability conditioned with a weighted sum of rewards. This weighted sum of rewards is referred to as a value function and is updated according to the following rule:

$Q_{j}(t+1)=(1-\alpha) Q_{j}(t)+\alpha r_{j}^{\prime}(t)$.

$Q_{j}(t)$ is the value function for option $j$ at time $t . r_{j}^{\prime}(t) \in$ $\left\{r_{X}^{\prime}, r_{Y}^{\prime}\right\}$ is a biased reward at time $t$. For an unchosen option, $Q_{j}(t)$ is not updated. $\alpha \in[0,1]$ is a learning rate that specifies the extent to which the value function is updated.

As mentioned above, we assume that the decisionmaking of the players is stochastic. The probability of choosing an option $j \in\{X, Y\}$ given the value function is defined as follows:

$p\left(j \mid Q_{j}(t)\right)=\frac{\exp \left[\beta Q_{j}(t)\right]}{\sum_{k \in\{X, Y\}} \exp \left[\beta Q_{k}(t)\right]}$.

$\beta \in[0, \infty)$ is an exploration rate that controls the randomness of the choice of each player. If $\beta=0$, then the choice is uniformly random. As $\beta$ becomes closer to $\infty$, the choice becomes completely deterministic.

\section{Time-averaged frequency}

In the present study, we are interested in which efficient or inefficient social conventions are formed depending on the given parameter set of $\alpha, b$, and $\theta$. As an observable, we introduce the time-averaged frequency as follows:

$\overline{f_{X}}:=\frac{1}{T} \sum_{t=0}^{T-1} f_{X}(t)$

where $T$ is the computational time required to calculate the time average. By calculating $\overline{f_{X}}$ for each parameter set, we can evaluate how often both social conventions are formed under a given parameter set. If $\overline{f_{X}}$ is close to 1 in a certain parameter set, then an efficient social convention is likely to be formed, and vice versa. 
In this study, we set the number of players as $N=$ 100. The exploration rate $\beta$ was fixed at 30.0 and $Q_{j}(0)=$ 0 for all players. The computational time $T=1.0 \times 10^{3}$ and used 50 different initial conditions (initial frequencies $f_{X}(0)$ ) for each parameter set. According to $N=100$ and $Q_{j}(0)=0$ for all players, we can consider the initial frequencies $f_{X}(0)=\{0.0,0.01, \ldots, 1.0\}$ follow binomial distribution $B(n, p)$ with $n=100, p=0.5$.

\section{RESULTS}

\section{A. Formation of social convention}

Figure 2 shows the results of the numerical simulation. It is clearly observed that when the conformity bias coefficient $b$ is sufficiently large, an inefficient social convention can be formed for a broad area of the parameter region. For the case of $\theta=0.1$, an inefficient convention is formed with a probability of almost 0.5 if the conformity bias coefficient $b$ is higher than 0.6 (Figure 2, top). This tendency is more clearly observed when the relative "badness" of option $Y$ compared with $X$ is smaller, i.e., $\theta=0.5$ (Figure 2, bottom).

Focusing on the learning rate $\alpha$, we found that when $\alpha$ is sufficiently low, an efficient convention is likely to be formed. On the other hand, the higher the value of $\alpha$, the more inefficient the convention that can be formed. This result implies that if the individual trial-and-error learning is slow enough (small $\alpha$ ), then learning can overcome the conformity bias, which can hinder the formation of an efficient convention.

\section{B. Tipping points}

Next, we analyzed the dynamics of the model. When analyzing the dynamics of formation of the convention, the most important point is whether the tipping points exist. Here, the tipping point $f_{X}^{*}$ is defined as the frequency of option $X$, as follows:

$$
f_{X}^{*}=\left\{\begin{array}{l}
\lim _{t \rightarrow \infty} f_{X}(t)=1, f_{X}(0) \geq f_{X}^{*} \\
\lim _{t \rightarrow \infty} f_{X}(t)=0, f_{X}(0)<f_{X}^{*}
\end{array}\right.
$$

In other words, the tipping point is a threshold that divides the dynamics into one converging to the efficient and the other converging to the inefficient convention. In Fig. 3, we show the typical dynamics of our model. These two typical dynamics are calculated with the same parameter sets and only differ in the initial frequency
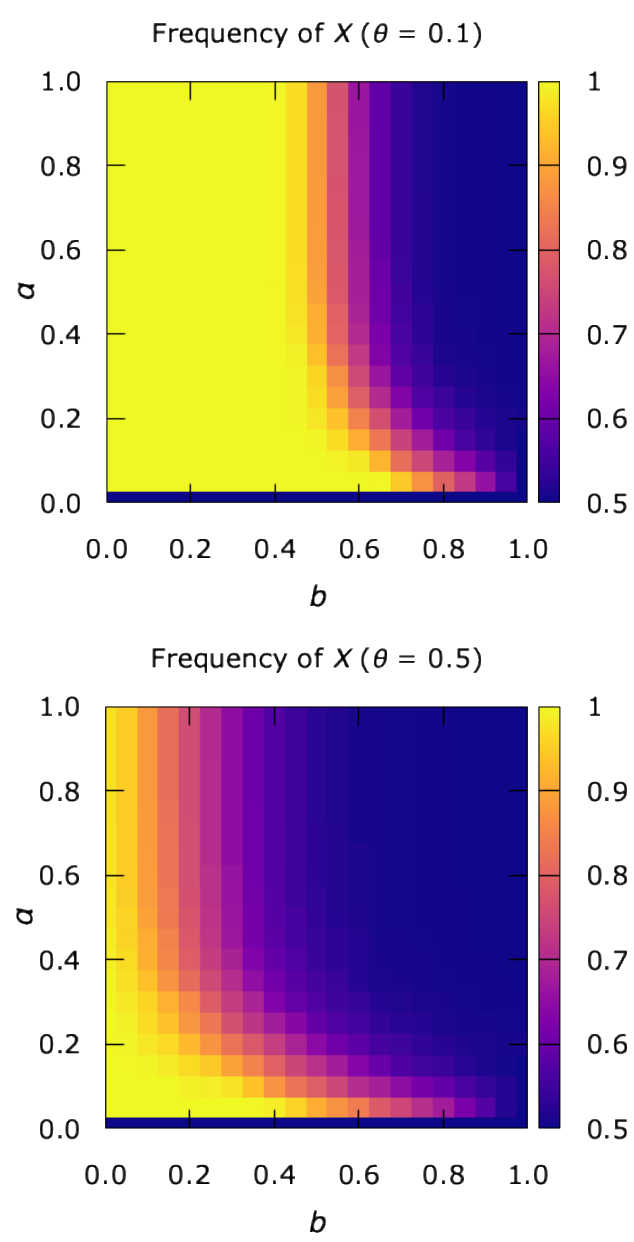

Fig. 2. Time-averaged frequency of $X$. For $\theta=0.1$ (top) and $\theta$ $=0.5$ (bottom). Each horizontal axis and vertical axis represent conformity bias $b$ and learning rate $\alpha$, respectively.

$f_{X}(0)$. With these examples, we can expect that there are some tipping points in our model.

The tipping points can be approximately derived from the reward function of the biased reward using Equations (6) and (7), where the frequency of option $X$ derived as the intersection of $r_{X}^{\prime}(t)$ and $r_{Y}^{\prime}(t)$ gives tipping points $f_{X}^{*}$. When $f_{X}(0)$ is larger than this frequency, option $X$ gives a higher reward than option $Y$. On the other hand, when $f_{X}(0)$ is smaller than this frequency, option $Y$ gives a higher reward than option $X$. Therefore, this frequency gives tipping points. Then, we obtain the tipping points $f_{X}^{*}$ as the frequency satisfying $r_{X}^{\prime}(t)=$ $r_{Y}^{\prime}(t)$; 
Time series
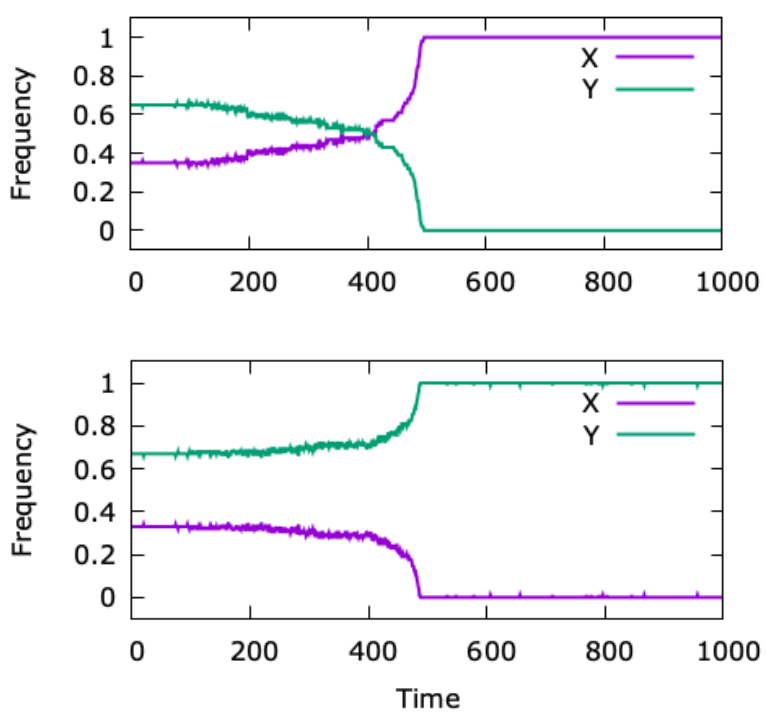

Fig. 3. Typical dynamics. Parameters: $\alpha=0.5, \beta=22.0, b=$ $0.5, \theta=0.1$. Initial frequency $f_{X}(0)=0.35$ (top) and 0.33 (bottom).

$f_{X}^{*}=\frac{\theta(1-b)+b}{1+\theta(1-b)+b}$.

As can be seen, $f_{X}^{*}$ monotonically increases given $\theta$. In Figure 4 , we show $f_{X}^{*}$ for various $\theta$ values.

However, it should be noted that Equation (12) does not include learning parameters $\alpha$ and $\beta$ because $r_{X}^{\prime}(t)$ and $r_{Y}^{\prime}(t)$ are just reward functions that do not consider the learning of the players. Hence, Equation (12) should be considered as an approximation of the tipping points with the case involving individual learning. In other words, we can use Equation (12) as a baseline for the case with individual learning.

To investigate the tipping points with the case involving individual learning, we analyzed the stationary states by using the initial frequency $f_{X}(0)$ as a parameter. Given an initial frequency, if the dynamics converge to $f_{X}(t)=1.0$, we found that the initial frequency exceeds the tipping point at given parameter sets, and vice versa.

Using the results of the numerical analysis shown in Figure 5, we can easily find that there are tipping points. Given conformity bias $b$, when increasing the initial frequency $f_{X}(0)$ from 0.0 to 1.0 (vertical direction in the figures), the dynamics starting from $f_{X}(0)$ that exceed a certain point converge to $f_{X}(t)=1.0$. In Figure 5 , we represent a set of points as a blue line.

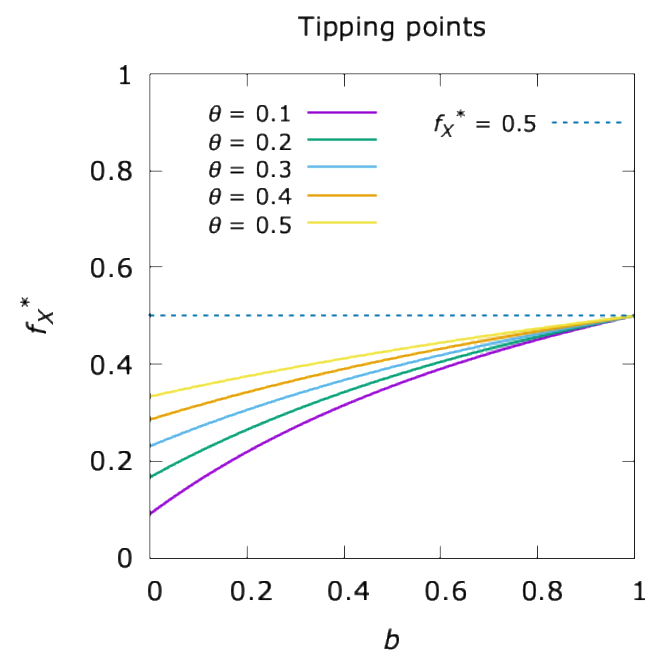

Fig. 4. Tipping points derived with theoretical analysis via Equation (12). Each horizontal axis and vertical axis represents conformity bias $b$ and initial frequency $f_{X}(0)$.

We also found a clear difference between the tipping points for the cases with and without individual learning. If the conformity bias is sufficiently large, then the tipping points for the case with the learning exceed those without the learning (i.e., the blue line exceeds the green line in the figure). In other words, an individual's trialand-error learning hinders the formation of an efficient convention. This characteristic is commonly observed in the results for both $\theta=0.1$ and 0.5 .

By contrast, when the conformity bias is relatively low, how individual learning affects the formation of the convention depends on $\theta$. For a low $\theta$, the tipping points for the case with learning are lower than those without the learning (Figure 5, top). This means that an individual's trial-and-error learning can promote the formation of an efficient convention. However, this does not hold for a case with a higher $\theta$ (Figure 5, bottom). In this case, individual learning no longer promotes the formation of an efficient convention.

As we can see from the analyses here, the conformity bias and the individual's trial-and-error learning contribute to the formation of the convention while interacting with each other. Furthermore, the relative "badness" of the option $Y, \theta$, may play a crucial role whether the individual's learning promotes or hinders the formation of an efficient convention. 

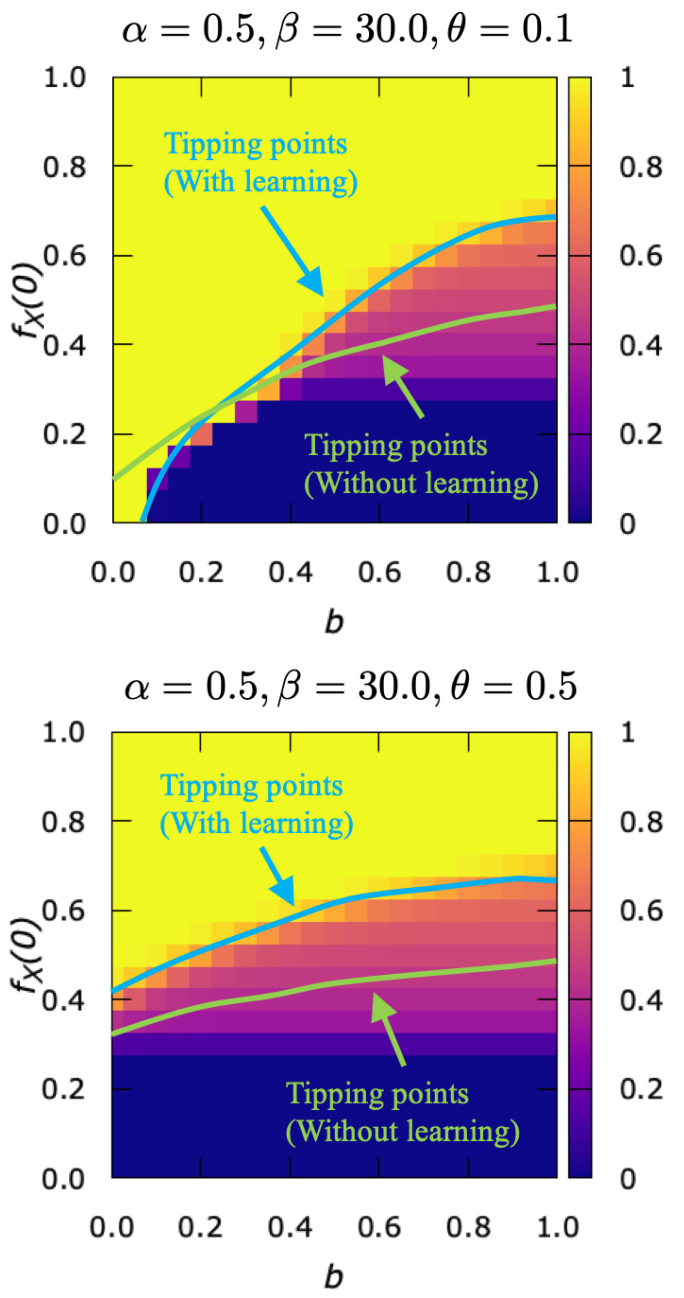

Fig. 5. Numerically calculated tipping points with individual learning for $\theta=0.1$ (top) and $\theta=0.5$ (bottom). Each horizontal axis and vertical axis represents conformity bias $b$ and initial frequency $f_{X}(0)$. Green line indicates theoretically derived tipping points per Equation (12). Blue line represents tipping points calculated numerically.

\section{DISCUSSION}

In the present study, we investigated the impact of conformity bias and an individual's trial-and-error learning on the formation of the convention. In analyzing stationary states, as the extent of conformity bias increases, an inefficient convention is likely to be formed. Even if the conformity bias is relatively high, the individual's trial-and-error learning can promote the formation of an efficient convention if the learning rate $\alpha$ is sufficiently small.
As a future direction, considering heterogeneous biases is an interesting and important issue that needs to be explored. We assumed that the conformity bias for each player was homogeneous in the present study. The bias of each player, however, varies in general, and the dynamics and an outcome emerging from interactions between these players can be more complex than in the homogeneous case. We need to analyze such a complex situation to understand the formation of the convention.

\section{CONCLUSION}

In this study, we investigated the impact of conformity bias and individual trial-and-error learning modeled with reinforcement learning on the formation of social conventions. We showed that conformity bias can drive the formation of both efficient and inefficient social conventions depending on the extent of the bias. An individual's trial-and-error learning can promote the formation of efficient conventions and overcome conformity bias. Further analysis of the mutual effect of conformity bias and individual learning will be performed in the future and reported elsewhere.

\section{ACKNOWLEDGEMENTS}

This study was supported by JSPS KAKENHI (Grant Number JP16H06397).

\section{REFERENCES}

[1] H.P. Young, "The evolution of social norms," Economics, vol. 7, no. 1, pp. 359-387, 2015.

[2] A. Baronchelli, "The emergence of consensus: a primer," Royal Society Open Science, vol. 5, no. 2, p. 172189, 2018.

[3] K. Nyborg, J. M. Anderies, A. Dannenberg, T. Lindahl, C. Schill, M. Schlu ter, W. N. Adger, K. J. Arrow, S. Barrett, S. Carpenter, et al., "Social norms as solutions," Science, vol. 354, no. 6308, pp. 42-43, 2016.

[4] [4] E. L. Paluck, H. Shepherd, and P. M. Aronow, "Changing climates of conflict: A social network experiment in 56 schools," Proceedings of the National Academy of Sciences, vol. 113, no. 3, pp. 566-571, 2016.

[5] G.L. Ciampaglia, A.Nematzadeh, F.Menczer, and A.Flammini, "How algorithmic popularity bias hinders or promotes quality," Scientific Reports, vol. 8, no. 1, pp. 17, 2018.

[6] M. B. Walker and M. G. Andrade, "Conformity in the asch task as a function of age," The Journal Of Social Psychology, vol. 136, no. 3, pp. 367-372, 1996. 
[7] M. Pasupathi, "Age differences in response to conformity pressure for emotional and nonemotional material.," Psychology and Aging, vol. 14, no. 1, p. 170, 1999.

[8] Brock, W. A., \& Durlauf, S. N. (2001). Discrete choice with social interactions. The Review of Economic Studies, 68(2), 235-260.

[9] Bentley, R. A., O'Brien, M. J., \& Brock, W. A. (2014). Mapping collective behavior in the big-data era. Behavioral and Brain Sciences, 37(1), 63.

[10] Caiado, C. C., Brock, W. A., Bentley, R. A., \& O’Brien, M. J. (2016). Fitness landscapes among many options under social influence. Journal of Theoretical Biology, 405, 5-16.

[11] Roth, A. E., \& Erev, I. (1995). Learning in extensive-form games: Experimental data and simple dynamic models in the intermediate term. Games and Economic Behavior, 8(1), 164-212.

[12] Erev, I., \& Roth, A. E. (1998). Predicting how people play games: Reinforcement learning in experimental games with unique, mixed strategy equilibria. American Economic Review, 848-881.

[13] Sutton, R. S., \& Barto, A. G. (1998). Reinforcement learning: an introduction Cambridge. MA: MIT Press. 